\title{
REFERENCE-SCALED AVERAGE BIOEQUIVALENCE STUDY OF PAROXETINE UNDER FED CONDITIONS: VALIDATION OF SAMPLE SIZE ESTIMATION BY BOOTSTRAPPING TECHNIQUE
}

\author{
CHANDRAKALA $\mathbf{V}^{1 *}$, UTPAL KUMAR SANKI ${ }^{2}$ \\ ${ }^{1}$ Department of Pharmaceutics, East Point College of Pharmacy, Avalahalli, Bengaluru, Karnataka, India. ${ }^{2}$ Department of Biostatistics, \\ ICON Plc, Bengaluru, Karnataka, India. Email: chandrakala52@gmail.com
}

Received: 01 February 2019, Revised and Accepted: 18 March 2019

\section{ABSTRACT}

Objective: New technique was adopted and validated to estimate pivotal sample size from the pilot study data and to establish bioequivalence (BE) of highly variable drugs (HVD), paroxetine, a novel controlled release (CR) matrix tablets utilized ghatti Gum as a rate controlling membrane, in human subject under fed conditions by reference scale design.

Methods: Bootstrapping technique was adopted to calculate the pivotal sample size from pilot study data for HVD paroxetine. The reliability and validation of the method were tested in a semi replicate three sequence (RRT, RTR, and TRR where T stand for test drug and R stand for reference drug) cross-over BE study in 24 healthy subjects under fed conditions.

Results: The ratio of the pharmacokinetic (PK) metric obtained from the bootstrapping technique after $\log$ transformation was 1.04 for $\mathrm{C}_{\text {max }}, 1.23$ for AUCT, and 1.21 for AUCI with corresponding power of the study which was greater than $80 \%$ from pilot study data simulation. The ratio of the PK metric obtained from the reference scaling design in the present study was 1.00 for $\mathrm{C}_{\max }, 1.21$ for AUCT, and 1.17 for AUCI. The upper limit of the $\mathrm{C}_{\max }$ AUCT, and AUCI at $95 \%$ confidence limit was $-0.143,-0.136$, and -0.17 , respectively.

Conclusion: The test paroxetine CR formulation was bioequivalent with reference drug under fed conditions. The technique used for estimation of the sample size in the pivotal study was found reliable, and bootstrapping technique plays an important role in calculating sample size where intrasubject variability was immaterial.

Keywords: Reference scaling, Bootstrapping techniques, Sample size, Bioequivalence, Controlled release.

(c) 2019 The Authors. Published by Innovare Academic Sciences Pvt Ltd. This is an open access article under the CC BY license (http://creativecommons. org/licenses/by/4. 0/) DOI: http://dx.doi.org/10.22159/ajpcr.2019.v12i5.32319

\section{INTRODUCTION}

Paroxetine hydrogen chloride (paroxetine $[\mathrm{HCl}]$ ) is an antidepressant and belongs to the class of selective serotonin reuptake inhibitors (SSRIs) [1,2]. Paroxetine has the highest known affinity for the serotonin transporter (0.13 nanomoles) of any currently used antidepressant [3]. Paroxetine was previously reviewed as an antidepressant drug in 1991 and reported some amount of hepatic toxicity following the chronic use of the drug $[4,5]$. Paroxetine $(\mathrm{HCl})$ is now used to treat obsessivecompulsive disorders and panic disorders [6,7]. Paroxetine $(\mathrm{HCl})$ is a phenylpiperidine derivative and (-) enantiomer is optically active, used clinically $[8,9]$. Paroxetine $(\mathrm{HCl})$ is not available in any combination preparations. The reason was to reduce acute toxicity and improved tolerability of SSRI compared to previously frequently used tri- and tetracyclic antidepressants [10,11]. Furthermore, controlled release (CR) formulation of paroxetine is highly safe and tolerable compared to immediate release formulation [12].

Controlled clinical trial on pharmacokinetics (PK) of paroxetine exhibited high variability within the subjects $(>30 \%$ reference variability within subjects). The substantial change in the innovator formulation necessitates bioequivalence (BE) study as per scale-up and post-approval changes (SUPAC) guideline (FDA, Guideline for industry, 1997) for registration of the generic product [13]. FDA proposed scaled average BE (SABE) study in 2008 to establish BE of highly variable drugs (HVD) with a minimum subject of $24[14,15]$. The most important feature of the SABE study is that a fixed sample size is adequate to demonstrate BE regardless of within-subject variability. In the present study, bootstrap technique is used to fix sample size and to calculate test/reference (T/R) ratio and power of the study from pilot data. Hence, the aim of the current research was to estimate sample size calculation and validation by bootstrapping techniques from the pilot study. The other aim of the study was to establish BE of paroxetine $37.5 \mathrm{mg}$ CR tablets by adopting three-period, three-sequence, two-treatment reference replicate (SABE) proposed by regulatory agency [16].

\section{MATERIALS AND METHODS}

Materials

Paroxetine and fluoxetine were obtained from Lupin Bioresearch Center, Mumbai, India. Acetonitrile, n-hexane, and methanol were procured from Sigma-Aldrich, Bengaluru, India. Analytical grade formic acid, amyl alcohol, and sodium hydroxide were purchased from local chemical store. Water was purified using in-house Milli-Q-System. All chemicals used for method development and validation were of analytical grade.

The liquid chromatography (LC) system used was API4000 AB SCIEX (California, USA) LC system equipped with triple-quadrupole mass analyzers and XBridge OST (BEH) C 18 column ( $50 \mathrm{~mm} \times 4.6 \mathrm{~mm}, 2.5 \mu$ particle size with a pore size of $130 \mathrm{~A}^{0}$ ). A Phenomenex SecurityGuard column (Bester, $4.0 \times 2.0 \mathrm{~mm}$ ) was used to protect the original column.

\section{Methods}

\section{Formulation development}

Extended-release (ER) matrix tablets were prepared by wet granulation technique [17]. The exact quantity of drug and inactive ingredients (Table 1) was passed through sieve \#40 and mixed thoroughly in octagon blender. The blend was mixed with isopropyl alcohol to make a dough mass which was then transferred to rapid mixture granulator to get wet granules. The wet granules were dried at room temperature 
$70^{\circ} \mathrm{C}$ for about $15 \mathrm{~min}$. The dried granules were then passed through sieve no 20. The extra-granular lubricant talc was mixed with granules and finally punched by a 27 station rotary tablet punching machine. The list of inactive ingredients used for the formulation of the tablets is given in Table 1.

High-performance LC method development for the quantification of paroxetine in human plasma

Various methods have already been established to quantify paroxetine in biological fluids [18-29]. Such methods include gas chromatography (GC) with nitrogen and mass spectrometry (MS) detection or simultaneous estimation with the same chemical category compounds by LC-tandem mass spectrometry (LC-MS/MS) or GC-MS or LC with voltammetric detection or LC with ultraviolet or MS analysis. However, in the present study, low solvent consuming and best recovery MS analysis method was applied to quantify picogram level of drug concentration in human plasma. The method was validated with respect to liquid-liquid extraction, reversed-phase LC, and MS/MS detection to perform the selective determination of paroxetine. Tandem MS was selected to improve the selectivity and sensitivity of the method of determination. The LC conditions, composition of mobile phase, and the MS/MS optimization were investigated to select the most appropriate operating conditions. The validation of the method was performed with respect to parameters such as linearity of the chromatographic response, precision, and accuracy which meets the accepted criteria for bioanalytical method validation and employed in BE study of two paroxetine $37.5 \mathrm{mg}$ ER tablets.

\section{Preparation of calibration curve}

A stock solution of paroxetine was prepared in methanol to obtain a concentration of $1000 \mu \mathrm{g} / \mathrm{L}$. An aliquot of the solution was evaporated to

Table 1: List of inactive ingredients used for the formulation of tablets

\begin{tabular}{|c|c|}
\hline Test drug & Reference drug \\
\hline Hypromellose & Hypromellose \\
\hline Lactose monohydrate & Lactose monohydrate \\
\hline Ghatti gum & Polyethylene glycol \\
\hline Purified talc & Purified talc \\
\hline $\begin{array}{l}\text { FD and C yellow No. } 6 \\
\text { aluminum lake }\end{array}$ & FD and C yellow No. 6 aluminum lake \\
\hline $\begin{array}{l}\text { D and C yellow No. } 10 \\
\text { aluminum lake }\end{array}$ & D and C yellow No. 10 aluminum lake \\
\hline Ghatti gum & Polymethyl methacrylate \\
\hline Polysorbate 80 & Polysorbate 80 \\
\hline Sodium lauryl sulfate & Sodium lauryl sulfate \\
\hline Titanium dioxide & Titanium dioxide \\
\hline
\end{tabular}

dryness and reconstituted with blank plasma and vortexed for 15 min to get a final concentration of $1 \mu \mathrm{g} / \mathrm{L}$. From this solution, eight calibration standard solutions containing 200, 500, 1000, 2000, 4000, 6000, 8000, and $10,000 \mathrm{ng} / \mathrm{L}$ and three quality control solutions at concentrations of 500,5000 , and $10,000 \mathrm{ng} / \mathrm{L}$ were prepared in blank plasma. The stock solution of fluoxetine was prepared in methanol and aliquot of the solution was evaporating to dryness and used as internal standard (IS). The dried IS was reconstituted with blank plasma and vortexed for $15 \mathrm{~min}$ to get a final concentration of $1 \mu \mathrm{g} / \mathrm{L}$. The calibration curve of paroxetine in blank human plasma and fluoxetine as IS is given in Fig. 1.

\section{Preparation of plasma sample for analysis}

Extraction of paroxetine from human plasma sample was carried out following addition of $100 \mathrm{mg} \mathrm{NaCl}, 200 \mu \mathrm{l}$ of $0.1 \mathrm{M} \mathrm{NaOH}, 5 \mathrm{ml}$ hexane:isoamyl alcohol $(99: 1, \mathrm{v} / \mathrm{v})$, and $20 \mu \mathrm{L}(1 \mu \mathrm{g} / \mathrm{L})$ of IS to $0.5 \mathrm{ml}$ plasma sample. The mixture was vortexed for $15 \mathrm{~min}$ and centrifuged at $3000 \mathrm{rpm}$ for $15 \mathrm{~min}$ at $4^{\circ} \mathrm{C}$ to separate an organic phase. $5 \mathrm{~mL}$ of the supernatant solution was transferred to $10 \mathrm{ml}$ Eppendorf vial and evaporated to dryness after addition of $50 \mu \mathrm{L}$ of $1 \mathrm{~N}$ HCL. The dried mixture was reconstituted with mobile phase (solution of formic acid [0.1\%] in acetonitrile:water [6:4 $\mathrm{v} / \mathrm{v}]$ pumped at a flow rate of $0.15 \mathrm{~mL} / \mathrm{min}$ ).

\section{Validation of the method}

The recovery of paroxetine was determined in three different QC samples having concentrations of 500,5000, and 10,000 ng/L in blank blood plasma. Plasma samples with the added drug at the concentrations specified above were extracted in triplicate. The concentrations of these samples were calculated on the basis of calibration curves constructed from the data for the drug not subjected to the extraction procedure.

To determine the intraassay precision, aliquots $(\mathrm{n}=6)$ of blank plasma containing the standard solution of paroxetine at concentrations of 500,5000 , and 10,000 ng/L were analyzed by the method proposed. To determine the interassay precision, blank plasma containing standard solution at the specified concentration was analyzed on 5 consecutive days. Linearity was obtained by analyzing control plasma samples $(n=6)$ containing standard solutions of paroxetine at concentrations of 500$10,000 \mathrm{ng} / \mathrm{L}$. The concentration range was estimated on the basis of the regression curve $\left(Y=1.004 \mathrm{X}+0.0045, \mathrm{R}^{2}=0.999\right)$ (Fig. 1) and correlation coefficient. The limit of quantification was determined by analyzing blank plasma samples enriched with decreasing concentrations of paroxetine standard solution. The limit of quantification was considered to be the lowest concentration quantified with an error of $<10 \%$ for a sample size of 5 . The selectivity of the method was evaluated by analyzing several drugs combined with paroxetine. The drugs that presented retention times close to those of the drugs under the study were submitted to the extraction procedure and rechromatographed.

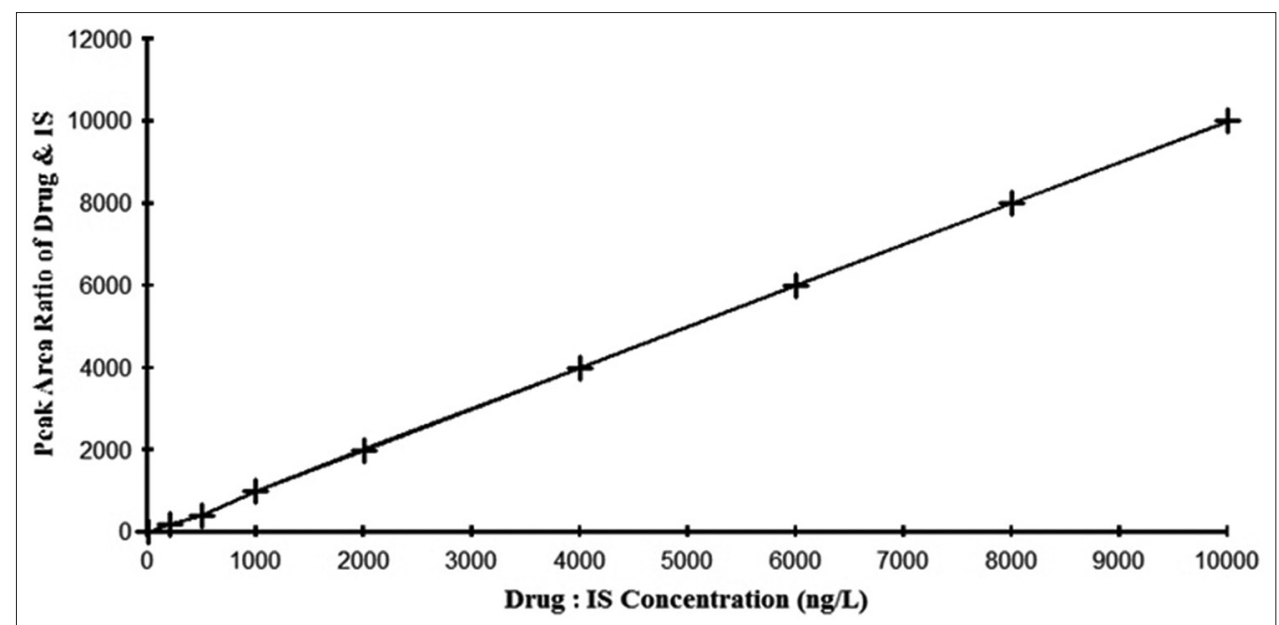

Fig. 1: Calibration curve of the paroxetine in blank human plasma using fluoxetine as internal standard in MRM mode $\left(Y=1.004 X+0.0045, R^{2}=0.999\right)$ 
BE study

Twenty-six healthy male Indian volunteers aged between 18 and 45 years with body mass index (BMI) within $19-29.4 \mathrm{~kg} / \mathrm{m}^{2}$ were enrolled for the study after assessment of their health status by general physical examination and clinical laboratory evaluation (including electrocardiogram [ECG] and chest X-ray). The normal value range of the following laboratory tests: Albumin, alkaline phosphatase, aspartate aminotransferase (AST), aminotransferase (ALT), blood glucose, creatinine, $\mu$-GT, total bilirubin, total protein, triglyceride, total cholesterol, hemoglobin, hematocrit, total and differential white cell counts, routine urinalysis, and negative serology for HIV, HBV, and HCV were determined before enrolling them in the study. All the subjects gave written informed consent, and the Madras Ethics Committee, Chennai, Tamil Nadu, India, approved the clinical study protocol. The study was conducted in accordance with ICH GCP, Indian GCP, ICMR guideline with the provisions of the Declaration of Helsinki (Seoul 2008).

The study was an open-labeled, randomized, three-sequence, threeperiod, single-dose, reference replicated three-way crossover design with 14 days' washout period between the doses. During each period, the volunteers were housed in a clinical pharmacology unit of Huclin Research Limited, Chennai, Tamil Nadu, India, on the eve of the dosing day. Following overnight fasting of about $10 \mathrm{~h}$ a high calorie, high fat breakfast was served 30 min before the study drug administration. A single dose of paroxetine (37.5 mg CR tablets of test or reference either formulation) was administered orally to each of the volunteers as per randomization code list at sitting posture with the aid of $240 \mathrm{ml}$ of water in a staggered manner to easy sample collection. A mouth check following drug administration, with the help of a tongue depressor, was performed for each of the volunteers to ensure the compliance of the dosing activities. All the subjects were restricted to $2 \mathrm{~h}$ post-drug administration water intake and toileting, while at other time, water was given ad libitum. A standard meal was provided to all the volunteers at 4,8 , and $12 \mathrm{~h}$ post-drug administration. Vitals were recorded at 2,4 , $6,8,12$, and $24 \mathrm{~h}$ post-drug administration for each volunteer to assess any health-related complication. No other food was permitted during the "in-house." Serial blood samples were collected from each of the volunteers at every period at pre-dose and at $04.00,06.00,08.00,10.00$, $12.00,14.00,16.00,18.00,20.00,24.00,28.00,36.00,48.0072 .00$, and $96.00 \mathrm{~h}$ post-drug administration.

\section{Sample size estimation for pivotal study}

A pilot study was conducted among 12 subjects in three-period, threesequence, cross-over design under fed conditions to establish sample size in the pivotal study. The within-subject reference variability of the area under the paroxetine plasma concentration-time curve from time 0 to the last measurable concentration (AUCT) and maximum drug concentration $\left(\mathrm{C}_{\max }\right)$ was $>0.3$ and the $\mathrm{T} / \mathrm{R}$ ratio of the drug was 1.27 and 1.3 , respectively. Based on the result of the pilot study, 6 test and 12 reference subject data were randomly selected from the pool of 36 individual subject data for all the three periods. The resultant 18 (1:2) subjects were considered as a boot sample and reanalysis the data to find the $\mathrm{T} / \mathrm{R}$ ratio then simulate to $36,45,54,60,72,90$ and 108 boot samples and then recalculate the $\mathrm{T} / \mathrm{R}$ ratio; this procedure was continued until $\mathrm{T} / \mathrm{R}$ ratio was within $0.80-$ 1.25. The simulation which produces the $\mathrm{T} / \mathrm{R}$ ratio within the limit of 0.8 to 1.25 was considered the final sample size for the pivotal study ignoring the subjects and sequence effect in the bootstrap analysis. To confirm the sample size, a power calculation was done further by resampling up to 1000 times from the boot sample and individual calculation of PK metric to find the ratio (Fig. 2).

Finally, the power was calculated based on the percentage of the time, and the ratio was within 80-125 from thousand bootstrapping [30-34]. If the power was failed to meet $80 \%$, then nearest boot sample was considered for further analysis to adjust the power of the study. In the present study, 72 boot samples were matched with the criteria of ratio between $80 \%$ and $125 \%$ which corresponds to a total number of subjects in the threeperiod design as $24(72 / 3)$. Hence, 24 subjects were included in the study; the bootstrapping simulation of the data of 72 samples produced the $\mathrm{T} / \mathrm{R}$ ratio of 1.23 for $\mathrm{C}_{\max }$ and 1.21 for AUCT after simulation.

\section{PK analysis}

Data from all subjects who completed all the three periods of the study were analyzed, and 24 subject data were used for PK and statistical analysis. The PK parameters of paroxetine were estimated using noncompartmental techniques [35]. The $\mathrm{C}_{\max }$ and the time elapsed to $\mathrm{C}_{\max }\left(\mathrm{T}_{\max }\right)$ were obtained directly from the data. The terminal-phase elimination rate constant was obtained using log-linear regression of the last four non-zero concentrations. The area under the paroxetine plasma concentration-time curve from time 0 to the last measurable concentration (AUCT) was computed using the linear trapezoidal rule. The area under the plasma concentration-time curve from time 0 to infinity (AUCI) was calculated as the sum of AUCT and $\mathrm{C}_{\mathrm{t}} / \mathrm{K}_{\mathrm{e}}$, where $C_{t}$ was the last measurable concentration. The elimination halflife was calculated as $\ln (2) / \mathrm{K}_{\mathrm{el}}$. All the PK analyses were performed by WinNonlin Professional, version 5.0.1 (Pharsight Corporation, Mountain View, California).

\section{Within subject reference variability}

From three-period designs, sequence term was used to extract reference treatment effect and residual mean square for calculating reference variability assuming reference crossed with reference as like of conventional two-way cross-over study [36,37]. BE testing of paroxetine was performed using a partial replicate three-way crossover design, and within-subject variability of primary PK parameters was calculated using mean square error (MSE) of log-transformed primary PK parameters (Equation 1).

Within subject reference variability $=(\sqrt{\operatorname{EXP}(M S E)-1}) \times 100$

The MSE was calculated by generalized linear modeling (GLM) procedure in SAS V 9.1.3 using sequence term to separate the cross-reference [38].

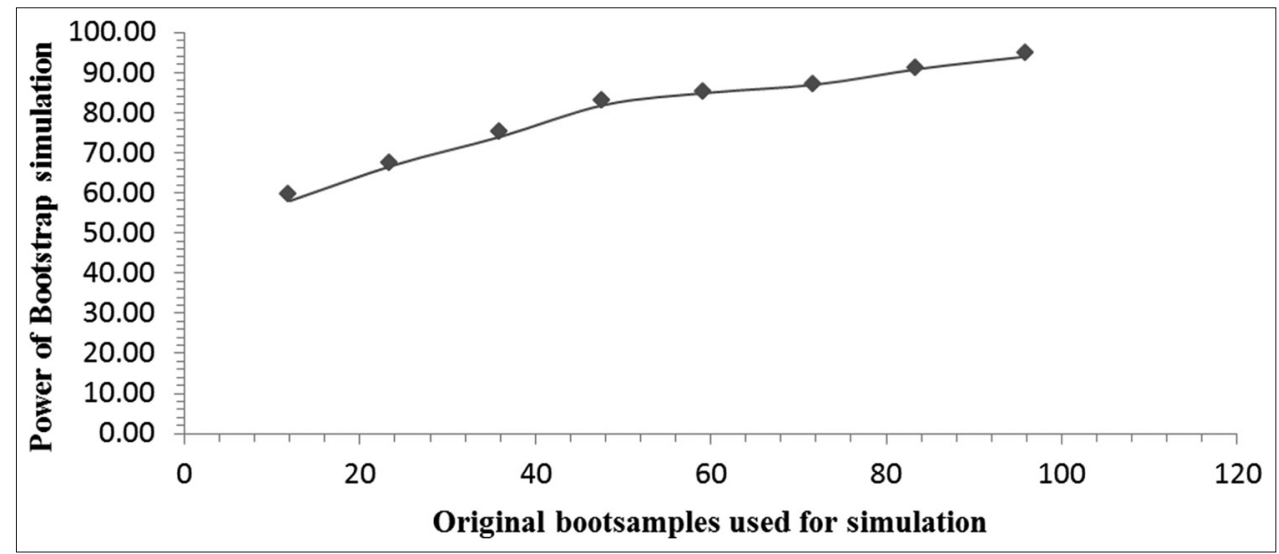

Fig. 2: Sample size and power estimation by 1000 boot samples analysis 
The within-subject variability of paroxetine was used as critical criteria for its identification as a HVD and was an important parameter for the application of SABE and population bioequivalence [39].

\section{Statistical analysis of PK data}

The data were analyzed for normal average BE as well as reference scale average $\mathrm{BE}$, and the statistical differences between the two approaches were evaluated. All the statistical analyses were performed by SAS V 9.2 (SAS Inc., Carry, USA). The primary PK parameters, namely AUCT AUCI, and $\mathrm{C}_{\text {max }}$, were log transformed to get parametric distribution, and logtransformed data were subjected to ANOVA analysis. A GLM procedure was used to evaluate formulation period and sequence effects on the primary PK parameters. The model included all primary PK parameters as main effect subjects nested within the sequence as random effect and sequence, period, and formulation as fixed effect [40]. The significance of all fixed effects was tested at alpha 0.05 and random effects at alpha $0.1[41,42]$.

Reference variability between subjects and within-subjects was calculated by the use of SAS program for reference scaled approach as prescribed by the FDA for establishing the bioavailability of HVD.

The ratio of all primary PK parameter such as AUCT, AUCI, and $\mathrm{C}_{\max }$ was also analyzed by the same program using PROC GLM model. The $90 \%$ confidence intervals (CIs) of the test/reference ratios for AUCI and $\mathrm{C}_{\max }$ were determined. Following national and international guidelines (including those of the FDA), the test and reference formulations were considered bioequivalent if the $90 \%$ CIs for the test/reference ratios of the log-transformed AUCI and $\mathrm{C}_{\text {max }}$ mean values were between 0.80 and 1.25 , and as per reference scaled approached point estimate of the ratio of PK parameters should fall within the 0.80-1.25 limit to show the BE as per FDA guidelines for HDV in a scale average BE approach and 95\% CI upper limit of the test to reference PK metric will be less than zero.

In addition, a non-parametric analysis (Wilcoxon signed-rank test) was applied to compare $\mathrm{T}_{\max }$ for both formulations. Non-parametric population characteristics were described with median and range.

\section{Statistical evaluation of average $\mathrm{BE}$}

Average BE (ABE) was applied for BE assessment of paroxetine in 24 healthy human male subjects. ABE was assessed using a two one-sided hypothesis testing procedure as follows [43].

$$
\mathrm{H}_{0}: \log (1.25) \leq\left(\mu_{\mathrm{T}}-\mu_{\mathrm{R}}\right) \leq \log (0.80)
$$

Versus

$$
\mathrm{H}_{1}: \log (0.8)<\left(\mu_{\mathrm{T}}-\mu_{\mathrm{R}}\right)<\log (1.25)
$$

Where $\mu \mathrm{T}$ and $\mu \mathrm{R}$ are the mean for test and reference formulations adjusted for period and sequence effects on a log-transformed scale, respectively. $\mathrm{ABE}$ was concluded as the $90 \% \mathrm{CI}$ for the ratio of test to reference limits within $0.80-1.25$ for all PK parameters.

\section{Statistical evaluation of SABE}

SABE is also considered as scaled individual BE. There is no subject by formulation interaction and the same within-subject variability of two drug products. Difficulties arise to establish BE for that drug product which fall under the categories of within-subject reference variability $\geq 30 \%$.

However, the scientists from FDA working group on HVD proposed the approach of reference SABER for the determination of BE in the case of HVD [44]. This method suggests that, if the expected intrasubject variability of a drug is $<30 \%$, the classic $0.80-1.25$ limits should be applied. However, for drugs with CVWR exceeding $30 \%$, a reference SABE approach should be used. These suggestions correspond to a switching coefficient of variation of $\mathrm{CVw} 0=30 \%$. It was proposed that three-period BE studies should be performed in which the reference product will be administered twice and the test product once, i.e., the possible sequences are TRR, RTR, and RRT. SABE was used to establish the $\mathrm{BE}$ of paroxetine using a minimal number $(\mathrm{n}=24)$ subjects. The formula used to expand the BE limit is given below of the SABER criterion as described by equation 4 .

$$
\text { Upper } / \text { Lower BE limits }=\operatorname{Exp}\left[ \pm \ln (1.25) * \frac{\mathrm{swr}}{\mathrm{swo}}\right]
$$

Where $s w r=$ Within-subject reference standard deviation (SD) and $s w o=$ Switching variability or regulatory limits.

SABE is concluded if the upper limit to the ratio of test to reference is zero or less than zero at $95 \% \mathrm{CI}$ and the GMR of this parameter is within $0.8-1.25$ to reduce the probability of type 1 error.

\section{RESULTS}

The generic paroxetine tablets were prepared using hypromellose, polyvinylpyrrolidone, ghatti gum, and glyceryl behenate. Polyethylene glycol and polymethyl methacrylate of the innovator formulation were replaced by ghatti gum. The list of other ingredients used in the development of the generic formula is given in Table 1. As per SUPAC guideline, changing one of the inactive ingredients from the innovator formula is considered to be major change. To obtain regulatory approval (USFDA) of the generic formulation with different inactive ingredients in comparison with reference listed drug require in vivo $\mathrm{BE}$ evaluation in human subjects. Hence, an in vivo BE study was conducted as per the FDA and EU guidelines to establish BE of developed generic formulation for marketing approval in the present study.

Twenty-six healthy adult male subjects were enrolled, 24 of whom completed all the periods of the study. The baseline demographic characteristics of the participants were expressed in terms of mean \pm SD (range): Age, 36.65 \pm 7.94 years (22-45); height, $169 \pm 12 \mathrm{~cm} \mathrm{(156-181);}$ and body weight, $58.34 \pm 4.37 \mathrm{~kg}(45-72)$. One subject withdrew due to abnormal results on liver function tests (described later) before period 3. This subject's liver function test results returned to normal within 1 month. One subject discontinued the study prematurely for reasons other than investigational drug-related adverse events or serious adverse events.

The mean PK parameters observed after the administration of the various regimens are summarized in Table 2 for reference drug and Table 3 for test drug.

The mean plasma concentrations after the administration of paroxetine CR tablet with water are shown in Figs. 3 and 4.

A short lag time in maximum absorption after the administration of paroxetine CR tablets was observed for test drug. However, there was no difference in PK parameters observed for both the formulation.

Treatment-emergent adverse events were reported by $45.8 \%$ of the subjects $(11 / 24)$ dosed during the study. Most of the adverse events were mild. Headache was the most common adverse event and was reported by $9.1 \%$ of subjects $(2 / 22)$ with regimen $A, 20.0 \%$ of subjects $(4 / 20)$ with regimen $B, 21.7 \%$ of subjects $(5 / 23)$ with regimen $C$, and $20.0 \%$ of subjects $(4 / 20)$ with regimen D. Before dosing in period 4 , one subject was found to have abnormal liver function test results (AST, $270 \mathrm{U} / \mathrm{L}$; alanine ALT, $875 \mathrm{U} / \mathrm{L}$; lactate dehydrogenase, $658 \mathrm{U} / \mathrm{L}$; and alkaline phosphatase, $252 \mathrm{U} / \mathrm{L}$ ). At the final follow-up assessment, all levels had returned to within their respective normal ranges.

The linear mean plasma concentration versus time curves of test and reference paroxetine formulations to the 24 subjects under fed condition are given in Figs. 3 and 4, respectively. The primary and secondary PK parameters for both the formulations under fed condition are tabulated in Tables 2 and 3. The descriptive statistics for untransformed PK parameters for both the formulations under fed 
Table 2: Descriptive statistics of pharmacokinetic parameters after reference treatment (fed conditions) (n=48)

\begin{tabular}{|c|c|c|c|c|c|c|}
\hline Statistics & $\mathbf{K}_{\mathrm{el}}\left(\mathbf{h}^{-1}\right)$ & $T_{\text {half }}(\mathbf{h})$ & $\mathbf{T}_{\max }(\mathbf{h})$ & $\mathrm{C}_{\max }(\mathrm{ng} / \mathrm{L})$ & AUCT (ng/L*h) & AUCI (ng/mL*h) \\
\hline Mean & 0.046 & 18.376 & 17.083 & 9701.246 & 297101.712 & 339057.078 \\
\hline SD & 0.017 & 10.595 & 6.672 & 8266.247 & 267131.434 & 338282.858 \\
\hline Minimum & 0.01 & 9.71 & 10.00 & 761.96 & 10630.02 & 11676.93 \\
\hline Median & 0.05 & 13.26 & 15.00 & 7383.74 & 215353.70 & 218855.63 \\
\hline Maximum & 0.07 & 52.65 & 28.00 & 35161.33 & 984853.59 & 1376373.59 \\
\hline $\mathrm{CV} \%$ & 37.3 & 57.7 & 39.1 & 85.2 & 89.9 & 99.8 \\
\hline Geometric mean & 0.042 & 16.359 & 15.943 & 6635.088 & 179606.618 & 196337.926 \\
\hline 95\% CI upper & 0.082 & 40.293 & 30.885 & 26801.281 & 849705.187 & 1038848.486 \\
\hline
\end{tabular}

SD: Standard deviation, CI: Confidence interval, CV: Coefficient of variation

Table 3: Individual subject pharmacokinetics parameters after test treatment (fed conditions) $(n=24)$

\begin{tabular}{|c|c|c|c|c|c|c|}
\hline Statics & $K_{e l}\left(h^{-1}\right)$ & $\mathbf{T}_{\text {half }}(\mathbf{h})$ & $\mathbf{T}_{\max }(\mathbf{h})$ & $\mathrm{C}_{\max }(\mathrm{ng} / \mathrm{L})$ & AUCT (ng/L*h) & AUCI (ng/L*h) \\
\hline Mean & 0.050 & 16.576 & 17.667 & 8195.932 & 272185.417 & 290550.049 \\
\hline SD & 0.021 & 7.785 & 5.710 & 5091.449 & 145972.357 & 166821.804 \\
\hline Minimum & 0.02 & 7.09 & 10.00 & 1046.68 & 23826.81 & 25542.89 \\
\hline Median & 0.05 & 14.45 & 17.00 & 7171.07 & 258468.09 & 273805.94 \\
\hline Maximum & 0.10 & 35.32 & 28.00 & 20242.99 & 534277.73 & 644989.53 \\
\hline CV\% & 41.7 & 47.0 & 32.3 & 62.1 & 53.6 & 57.4 \\
\hline Geometric mean & 0.046 & 15.178 & 16.880 & 6663.217 & 217943.875 & 229617.563 \\
\hline 95\% CI upper & 0.095 & 33.710 & 30.235 & 19402.135 & 593468.407 & 657722.364 \\
\hline
\end{tabular}

SD: Standard deviation, CI: Confidence interval, CV: Coefficient of variation

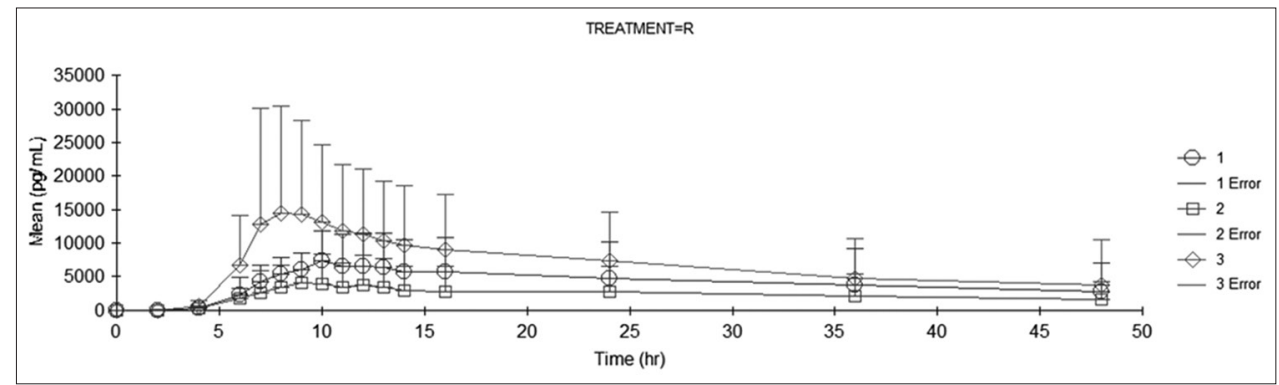

Fig. 3: Linear mean plasma concentration of paroxetine reference product at three different periods under fed conditions $(\mathrm{n}=48)$

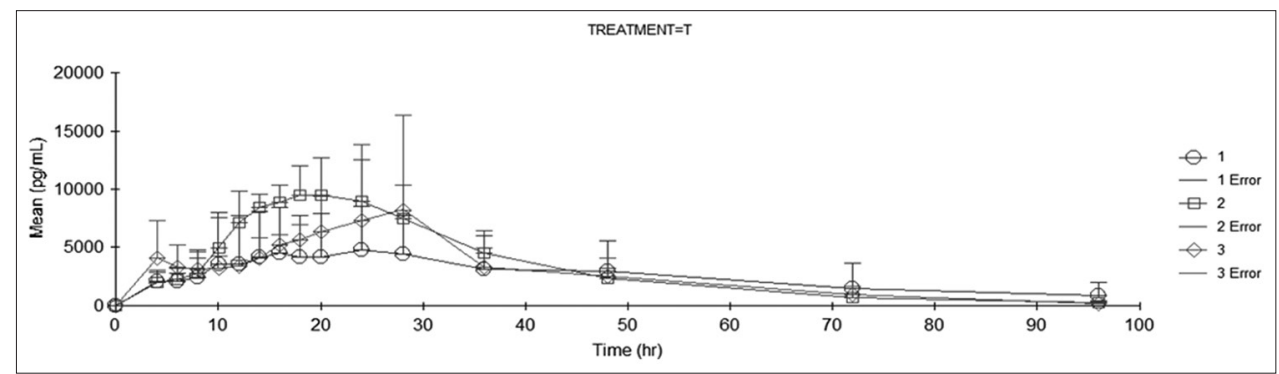

Fig. 4: Linear mean plasma concentration of paroxetine test product at three different periods under fed conditions ( $=24$ )

condition is presented in Tables 2 and 3, respectively. The mean $( \pm$ SD) $\mathrm{C}_{\text {max }}(\mathrm{ng} / \mathrm{L})$ of test and reference formulations is $8195.9( \pm 5091.5)$ and $9701.3( \pm 8266.3)$, respectively. The mean $( \pm \mathrm{SD})$ AUCT $\left(\mathrm{ng} / \mathrm{L}^{*} \mathrm{~h}\right)$ of test and reference is $272185( \pm 145972)$ and $297102( \pm 267131)$, while mean $( \pm \mathrm{SD})$ AUCI $\left(\mathrm{ng} / \mathrm{L}^{*} \mathrm{~h}\right)$ of test and two reference formulations is $290550( \pm 166822)$ and 339057 ( \pm 338283$)$, respectively. The mean $( \pm S D)$ of $T_{\max }(h), K_{e l}\left(h^{-1}\right)$, and $t_{\text {half }}(h)$ of test and reference formulations of paroxetine is $17.7 \mathrm{~h}( \pm 5.7)$ and $17.1 \mathrm{~h}( \pm 6.7), 0.05 \mathrm{~h}^{-1}( \pm 0.21)$ and $0.05 \mathrm{~h}^{-1}( \pm 0.17)$, and $11.0 \mathrm{~h}( \pm 27.8)$ and $18.4 \mathrm{~h}( \pm 10.6)$, respectively. The results of descriptive statistics of primary PK parameters of test and two reference products are presented in Tables 2 and 3, respectively. The reference variability (within-subject reference variability) of the primary PK parameters of $\mathrm{C}_{\text {max }}$ AUCT (AUClast), and AUCI (AUC total) is $0.58,0.72$, and 0.74 , respectively. Details are presented in Table 4 . The geometric mean ratio between two formulation and $95 \%$ CI for the primary PK parameters calculated from the ANOVA was 100\%, $-0.143113 ; 121 \%,-0.136539$; and $117 \%,-0.170342$, respectively (Table 4) meeting the criteria of scale average BE.

All the 24 subjects completed all the three periods of the study and there was no drop out or withdrawal. No serious adverse events were reported except abdominal pain in subject 01 and mild diarrhea in subject 02 . However, such complications were resolved without sequel. There were no clinically significant changes in vital signs, clinical laboratory variables, ECG, X-ray, and general physical examination. 
Table 4: Reference scaling of main PK parameters with 95\% confidence interval

\begin{tabular}{llllll}
\hline Parameter & $\mathbf{S}_{\mathrm{wR}}$ & Point estimate & Standard error & Degrees of freedom & 95\% upper confidence limit \\
\hline $\mathrm{C}_{\max }$ & 0.5808352 & 1.00 & 0.1137 & 21 & -0.143113 \\
AUCT & 0.7223151 & 1.21 & 0.1331 & 21 & -0.136539 \\
AUCI & 0.7358902 & 1.17 & 0.1338 & 21 & -0.170342 \\
\hline
\end{tabular}

\section{DISCUSSION}

The generic paroxetine $37.5 \mathrm{mg}$ ER tablets were prepared using hypromellose, polyvinylpyrrolidone, ghatti gum, and glyceryl behenate. Polyethylene glycol and polymethyl methacrylate in the innovator formulation were replaced by ghatti gum. The list of other ingredients used in the development of the formula is given in Table 1. The ghatti gum was used to serve as a rate-controlling membrane for the ER formulation. Due to the change of the inactive ingredients, a BE study was needed to get regulatory approval of the drug for marketing. Keeping this in the mind, BE study was conducted under fed conditions. The inherent nature of the molecule was to behave differently in in vivo while dosing at the same individual at different occasion. Such variability was observed for all the primary PK parameters (GSK clinical trial registry study identifier, PCR: 112811 and 112812). Due to high variability observed in the PK parameters, it was needed more number of subjects to establish BE between dosage forms by a traditional average BE in two-way cross-over design. To avoid exploiting a large number of healthy volunteers, a reference scale approach was adopted to establish BE. The reference scale utilizes the reference variability $>30 \%$; if a PK metric shows that it variability between reference is $<30 \%$, then conventional BE approach will be adopted; otherwise, $95 \% \mathrm{CI}$ test to reference ratio upper limit should be negative and test to reference ratio should be within 0.8-1.2 to claim BE. The method utilized two reference administration in the same subject at different periods and one test administration at another period; therefore, three-period, three-sequence, cross-over design was ideal to calculate reference variability.

This study was open-labeled, three-period, three-sequence, twotreatment cross-over design utilizing minimum subjects of 26 under fed conditions. A randomization was generated to eliminate the bias of selecting test and reference drug for subjects. The descriptive statistics of age, height, weight, and BMI of the 24 subjects participated in the study was within the limit of 18-45 years, 1.48$1.78 \mathrm{~m}, 55-75 \mathrm{~kg}$, and $19-29.4 \mathrm{Kg} / \mathrm{m}^{2}$, respectively. The drugs were dosed on the dosing day to the volunteers as per randomization code list $30 \mathrm{~min}$ after the completion of the high-fat high-calorie breakfast. The study-related procedure and blood collections were done at the scheduled time to quantify the plasma concentration of the drug. There were no reported serious adverse events, signifying that the Ghatti gum used for the development of the generic formulation is well tolerable in the human subject. Since the ghatti gum obtained from the natural sources, some microbial contamination will always be present after finest purification too. Therefore, it is obvious that there will be some amount of temperature rising following intake of the gum, but in our study, it was found that there were no such complications regarding fever to the subject which indicated the safety of the ghatti gum.

Both paroxetine and IS were extracted from plasma samples by solidphase extraction using OASIS HLB extraction plates. Then, the plasma samples were washed further with a water:methanol $(80: 20, \mathrm{v} / \mathrm{v})$ mixture. Mixtures containing varying percentages of methanol were tried. The 80:20 mixtures gave optimum results in terms of recovery and selectivity. The recovery of methanol:water mixture was not stable, and hence, the eluate was evaporated and the residue was reconstituted with mobile phase. A validated LC-MSMS method was used to quantify drug in the plasma samples. An eight-point calibration standard curve in human plasma, ranging from 200 to $10000 \mathrm{ng} / \mathrm{L}$ for paroxetine, was prepared in duplicate for each run. The standard curve was satisfactorily described by linear regression weighted by $1 / x$, as the back calculated values were all within $61.5 \%$ of the nominal concentrations $(62.0 \%$ at the LLOQ) in the three validation runs, which are not shown in the result. 26 of 24 enrolled subjects completed all the three periods of the study.

The PK profile of paroxetine, a single dose three-period, threesequence, two-treatment cross-over reference replicate study with a minimum wash-out period of 14 days, carried out in fed conditions was considered to be adequate. The linear mean $( \pm \mathrm{SD} n g / \mathrm{L})$ concentration versus time graph of the reference formulation in three different periods is given in Fig. 3 and of test in Fig. 4. The descriptive statistics of PK parameters of test and reference drug are given in Tables 2 and 3 , respectively. A test drug is considered to be pharmacokinetically equivalent, i.e., bioequivalent to a reference drug product if the $95 \%$ CI of the test and reference geometric mean ratios of the AUCs and $\mathrm{C}_{\max }$ is found to be zero or less than zero and point estimates are in the range of 0.8-1.2. The appropriateness of the reference scaling approach was established from the $S_{W R}$ values of the primary PK parameters $\left(\mathrm{C}_{\max }\right.$, AUCT, and AUCI which were 0.58, 0.74, and 0.78, respectively); established primary $\mathrm{PK}$ parameters from this study at 95\% CIs were within the pre-defined BE criteria (Table 4). The study results revealed that the two formulations of paroxetine were similar in PK characteristics among these healthy Indian subjects under fed conditions.

\section{CONCLUSION}

The sample size calculation for SABE from the pilot data using bootstrapping resampling techniques was adequate to estimate $\mathrm{T} / \mathrm{R}$ ratio with only $1 \%$ deviation from the observed data. The LC-MSMS method used for the analysis of plasma samples was reliable with a good reproducibility of the results. The reference scale semi-replicate three-period, three-sequence, two-treatment in vivo clinical study was adequate to establish the difference of their geometric mean for all the primary PK metric.

There were no serious adverse events of the study and no change in the abnormal laboratory value for the subjects except diarrhea to the subjects 20 and 18 in period two and subject 13 in period three which were mild in nature and resolved without sequel.

The assumption of reference scaling for the BE study is proved since the within-subject reference variability $\left(\mathrm{S}_{\mathrm{WR}}\right)$ for $\mathrm{C}_{\max }$ AUCT, and AUCI is 0.58, 0.72, and 0.73, respectively, under fed conditions; therefore, reference scaling approaches were used to establish BE and test product found $\mathrm{BE}$ with respect to the reference formulation, and hence, we can conclude that the ghatti gum can be used as rate-controlled membrane in certain concentration to develop CR formulation of other drugs.

\section{ACKNOWLEDGMENT}

The authors wish to thank Huclin Research Labs Pvt. Ltd., Chennai, India, for providing method validation and analysis of plasma samples. The authors also wish to thank Lupin Bioresearch Center, Mumbai, India, for procurement of IS and drugs.

\section{AUTHORS' CONTRIBUTION}

The authors were involved in study initiation, ethical committee approval, statistical and PK evaluation, and clinical study report. 


\section{CONFLICTS OF INTEREST}

We wish to confirm that there are no known conflicts of interest associated with this publication and there has been no significant financial support for this work that could have influenced its outcome.

We confirm that the manuscript has been read and approved by all named authors and that there are no other persons who satisfied the criteria for authorship but are not listed. We further confirm that the order of authors listed in the manuscript has been approved by all of us.

We further confirm that any aspect of the work covered in this manuscript that has involved either experimental animals or human patients has been conducted with the ethical approval of all relevant bodies and that such approvals are acknowledged within the manuscript.

We understand that the corresponding author is the sole contact for the editorial process (including editorial manager and direct communications with the office). He is responsible for communicating with the other authors about progress, submissions of revisions, and final approval of proofs.

\section{REFERENCES}

1. Baumann P. Pharmacokinetic-pharmacodynamic relationship of selective serotonin reuptake inhibitors. Clin Pharmacokinet 1996;31: 444-69.

2. Kaye CM, Haddock RE, Langley PF, Mellows G, Tasker TC, Zussman BD, et al. A review of the metabolism and pharmacokinetics of paroxetine in man. Acta Psychiatr Scand Suppl 1989;350:60-75.

3. Robert MN, Samuel TG, Bryman EW. Paroxetine-the antidepressant from hell? Probably not, but caution required. Psychopharmacol Bull 2016;46:77-104.

4. Benbow SJ, Gill G. Paroxetine hepatotoxicity. $\mathrm{Br}$ Med J 1997;314:1387-94

5. Caccia S. Metabolism of the newer antidepressants. Clin Pharmacokinet 1998;34:281-302.

6. Goldberg RJ. Antidepressant use in the elderly. Drugs Aging 1995;11:119-31.

7. Boyer WF, Feighner JP. An overview of paroxetine. J Clin Psychiatry 1992;53:3-6.

8. Eap CB, Baumann P. Analytical methods for the quantitative determination of selective serotonin reuptake inhibitors for therapeutic drug monitoring purposes in patients. J Chromatogr B 1996;686:51-63.

9. Dechant KL, Clissold SP. Paroxetine: A review of its pharmacodynamic and pharmacokinetic properties, and therapeutic potential in depressive illness. Drugs 1991;41:225-53.

10. Geretsegger G, Bohmer F, Ludwing M. Paroxetine in the elderly depressed patients: Randomized comparison with fluoxetine of efficacy, cognitive and behavioural effects. Int Clin Psychopharmacol 1994:9:25-9.

11. Gunasekara NS, Noble S, Benfield P. Paroxetine: An update of its pharmacology and therapeutic use in depression and a review of its use in other disorders. Drugs 1998;55:85-120.

12. Nemeroff CB. Paroxetine: An overview of the efficacy and safety of a new selective serotonin reuptake inhibitor in the treatment of depression. J Clin Psychopharmacol 1993;13:10-7.

13. Guidance for Industry, SUPAC-IR/MR: Immediate Release and Modified Release Solid Oral Dosage Forms U S Department of Health and Human Services, Food and Drug Administration, Center for Drug Evaluation and Research (CDER) 1997, Manufacturing Equipment Addendum, (Revision 1); 1999.

14. Guidance for Industry: Bioavailability and Bioequivalence Studies for Orally Administered Drug Products-General Gonsiderations (Revision 1). U S Department of Health and Human Services, Food and Drug Administration, Center for Drug Evaluation and Research (CDER); 2008.

15. The European Agency for the Evaluation of Medicinal Products, Human Medicines Evaluation Unit, International Conference on Harmonisation. Guideline for Good Clinical Practice [EMEAWeb site]. Available from: http://www.emea.eu.int. [Last accessed 2007 Mar 26].

16. European Medical Agency Guideline on the Investigation of Bioequivalence CPMP/EWP/QWP/1401/98 Rev. 1, London; 2010.

17. Reddy PS, Bose PS, Saritha D, Sruthi V. Formulation and evaluation of colon targeted matrix tablet using natural tree gums. Int J Pharm Pharm
Sci 2018;10:92-7.

18. Zhu Z, Neirinck L. High-performance liquid chromatography-mass spectrometry method for the determination of paroxetine in human plasma. J Chromatogr B Analyt Technol Biomed Life Sci 2002;780: 295-300

19. Maurer HH, Bickeboeller-Friedrich J. Screening procedure for detection of antidepressants of the selective serotonin reuptake inhibitor type and their metabolites in urine as part of a modified systematic toxicological analysis procedure using gas chromatography-mass spectrometry. J Anal Toxicol 2000;24:340-7.

20. Maralikova B, WeinmannW. Confirmatory analysis for drugs of abuse in plasma and urine by high-performance liquid chromatographytandem mass spectrometry with respect to criteria for compound identification. J Chromatogr B 2004;811:21-30.

21. Naidong W, Eerkes A. Development and validation of a hydrophilic interaction liquid chromatography-tandem mass spectrometric method for the analysis of paroxetine in human plasma. Biomed Chromatogr 2004; 18:28-36.

22. Eap CB, Bouchoux G, Amey M, Cochard N, Savary L, Baumann P. Simultaneous determination of human plasma levels of citalopram, paroxetine, sertraline and their metabolites by gas chromatographymass spectrometry. J Chromatogr Sci 1998;36:365-71.

23. Juan H, Zhiling Z, Huande L. Simultaneous determination of fluoxetine, citalopram, paroxetine, venlafaxine in plasma by high performance liquid chromatography-electrospray ionization mass spectrometry (HPLC-MS/ESI). J Chromatogr B Analyt Technol Biomed Life Sci 2005;820:33-9.

24. Erk N, Biryol I. Voltammetric and HPLC techniques for the determination of paroxetine hydrochloride. J Pharmazie 2003;58:699-704.

25. Duverneul C, de la Grandmaison GL, de Mazancourt P, Alvarez JC. A high-performance liquid chromatography method with photodiodearray UV detection for therapeutic drug monitoring of the nontricyclic antidepressant drugs. Ther Drug Monit 2005;25:565-73

26. Lopez-Calull C, Dominguez N. Determination of paroxetine in plasma by high-performance liquid chromatography for bioequivalence studies. J Chromatogr B Biomed Sci Appl 1999;724:393-8.

27. Bonato PS, Lanchote VL. A rapid procedure for the purification of biological samples to be analyzed by high-performance liquid chromatography. J Liq Chromatogr 1993;16:2299-308.

28. Martin AB, Dierdorf HD, Barry DZ, Peter EC. Determination of paroxetine in human plasma, using high-performance liquid chromatography with fluorescence detection. J Chromatogr 1987;419: 438-44.

29. Reddy GS, Reddy SL, Reddy SK. Development and validation of a stability indicating liquid chromatographic method for the simultaneous estimation of paroxetine and clonazepam in bulk and its pharmaceutical formulations. Int J Pharm Pharm Sci 2014;10:397-402.

30. Stephen JW, Michael JC. The use of bootstrap methods for estimating sample size and analyzing health-related quality of life outcomes. Stat Med 2005;24:1075-102.

31. Yuan KH, Hayashi K. Bootstrap approach to inference and power analysis based on three test statistics for covariance structure models. Br J Math Stat Psychol 2003;56:93-110.

32. Chow SC, Shao J, Wang H. Sample Size Calculation in Clinical Research. New York: Marcel Dekker Inc.; 2003.

33. Peng X, Peng G, Gonzales C. Power Analysis and Sample Size Estimation using Bootstrap, Paper sp05. Indianapolis, IN: Eli Lilly and Company.

34. Thompson D. Bootstrap Power Analysis using SAS ${ }^{\circledR+}$ Paper SA-13. Chicago, IL: Thompson Research Consulting LLC.

35. Shumaker RC. Pkcalc: A basic interactive computer program for statistical and pharmacokinetic analysis of data. Drug Metab Rev 1986;17:331-48

36. Tothfalusi L, Endrenyi L. Limits for the scaled average bioequivalence of highly variable drugs and drug products. Pharm Res 2010;20: 382-89.

37. Kamal KM, Maureen JR, John WH. Individual and average bioequivalence of highly variable drugs and drug products. J Pharm Sci 2000:86:1193-97.

38. Chow SC, Liu JP. Design and Analysis of Bioavailability and Bioequivalence Studies. New York: Marcel Dekker Inc.; 1992.

39. Baek I, Lee BY, Kang W, Kwon KI. Comparison of average, scaled average, and population bioequivalence methods for assessment of highly variable drugs: An experience with doxifluridine in beagle dogs. Eur J Pharm Sci 2010:39:175-80

40. Schuirmann DJ. A comparison of the two one-sided tests procedure and the power approach for assessing the equivalence of average 
bioavailability. J Pharmacokinet Biopharm 1987;15:657-80.

41. Tothfalusi L, Endrenyf L, Arieta AG. Evaluation of bioequivalence for highly variable drugs with scaled average bioequivalence. Clin Pharmacokinet 2009;48:725-43.

42. Sam HH, Makhlouf F, Donald JS, Hyslop T, Davit B, Conner D, et al. Evaluation of a scaling approach for the bioequivalence of highly variable drugs. AAPS J 2008;10:450-4.
43. Davit BM, Conner DP, Fabian-Fritsch B, Haidar SH, Jiang X, Patel DT, et al. Highly variable drugs: Observations from bioequivalence data submitted to the FDA for new generic drug applications. AAPS J 2008; 10:148-56

44. Haidar SH, Davit B, Chen ML, Conner D, Lee LM, Li QH, et al. Bioequivalence approaches for highly variable drugs and drug products. Pharm Res 2008;25:1237-41 